\title{
Evaluación microbiológica de dispositivos móviles en personal quirúrgico de una institución de salud, Pereira, Colombia, 2018
} \author{
2018 \\ German Oved Acevedo-Osorio 1* orcid.org/0000-0002-1499-2579 \\ Adriana Marcela Gómez-Fernández orcid.org/0000-0001-7055-6651 \\ Natalia Oyola-Leiva' orcid.org/0000-0002-5503-3307 \\ Laura Daniela Arboleda-Angulo' ${ }^{1}$ orcid.org/0000-0002-5561-8882 \\ Luisa María Orozco-Cardona' orcid.org/0000-0002-2672-9671
}

Microbiological analysis of mobile devices of surgical personnel working at a health care institution in Pereira, Colombia,

1 Fundación Universitaria del Área Andina - Seccional Pereira. Pereira, Colombia

Fecha de recepción: Mayo 19-2019 Fecha de revisión: Septiembre 10 - 2019 Fecha de aceptación: Diciembre 19 - 2019

Acevedo-Osorio GO, Gómez-Fernández AM, Oyola-Leiva N, Arboleda-Angulo LD, Orozco-Cardona LM. Evaluación microbiológica de dispositivos móviles en personal quirúrgico de una institución de salud, Pereira, Colombia, 2018. Univ. Salud. 2020;22(1):77-83. DOI: https://doi.org/10.22267/rus.202201.177

\section{Resumen}

Introducción: El uso del teléfono celular se ha vuelto común en áreas del hospital, incluida la sala de operaciones, aumentando el riesgo de contaminación cruzada y las altas tasas de infecciones del sitio quirúrgico. Objetivo: Determinar la contaminación microbiana en dispositivos móviles del personal quirúrgico de una Institución de Salud de Pereira, Colombia en el primer semestre del año 2018. Materiales y métodos: Estudio descriptivo observacional, transversal. Se tomaron 10 dispositivos móviles del personal del área de quirófano al azar y a cada uno se le realizó frotis con hisopo estéril al estuche o cobertura del celular. Las muestras se transportaron en tubos de ensayo con agua peptonada al $1 \%$ hasta el momento de la siembra; las muestras se analizaron por técnica microbiológica recuento en placa profunda. Resultados: En las muestras se encontró un promedio de 93 UFC (Unidades Formadoras de Colonias) de mesófilos aerobios, 13 UFC de coliformes totales, 22 UFC de mohos y levaduras. Conclusión: El análisis microbiológico permitió detectar la presencia de elevadas cantidades de unidades formadoras de colonias que podrían contribuir de manera significativa a incrementar las tasas en infecciones asociadas a la atención en salud.

Palabras clave: Teléfono celular; infección de la herida quirúrgica; análisis microbiológico; infección hospitalaria; desinfección. (Fuente: DeCS, Bireme).

\begin{abstract}
Introduction: Cell phone use in hospital areas such as surgery rooms has become a common practice, which has increased both the risk of cross-contamination and the rates of surgical site infections. Objective: To determine microbial contamination of mobile devices belonging to surgical staff of a Health Care Institution in Pereira, Colombia during the first semester of 2018. Materials and methods: An observational, cross-sectional, descriptive study was conducted with a sample of ten mobile devices randomly chosen from health personnel working in the surgical room. Surface samples were collected from cell phone cases and bags using sterile swabs that were were kept in a $1 \%$ peptone salt solution until a deep plate count assay was performed on them. Results: The plate count confirmed the presence of mesophilic aerobes (93 CFUs), total coliforms (13 CFUs) as well as yeasts and molds (22 CFUs). Conclusion: The microbiological analysis showed elevated numbers of colony-forming units that could significantly increase the rates of infections associated with health care settings.
\end{abstract}

Keywords: Cell phone; surgical wound infection; microbiological analysis; cross infection; disinfection. (Source: DeCS, Bireme). 


\section{Introducción}

Los dispositivos móviles se han convertido en uno de los accesorios más indispensables de la vida profesional y social. Se tornaron en el más importante medio de comunicación en todo el mundo, de fácil acceso, económico y fácil de usar. Son ampliamente utilizados por los trabajadores de la salud, pero con todos los logros y beneficios de dichos dispositivos, es fácil pasar por alto el peligro que representa para la salud de muchos pacientes en los ámbitos hospitalarios cuando no se manejan las medidas asépticas adecuadas(1).

El continuo contacto con los dispositivos móviles por parte de todos los usuarios hospitalarios como pacientes, visitantes y el distinto personal clínico, lo convierte en un fómite abierto y crítico para la transmisión de microorganismos, así como de infecciones asociadas a la atención en salud (IAAS) producto de las infecciones cruzadas, asociados con la humedad y temperatura optima del cuerpo humano presente en la piel, especialmente nuestras palmas. Estos factores y el calor generado por los teléfonos móviles contribuyen a albergar bacterias en el dispositivo a niveles alarmantes(2). A diferencia de las manos que se desinfectan comúnmente en las instituciones de salud, los dispositivos móviles no poseen un control exhaustivo y menos en procesos de desinfección o limpieza.

Varios estudios han podido identificar que alrededor del $90 \%$ de los dispositivos móviles del personal clínico son reservorios bacterianos susceptibles de causar infecciones intrahospitalarias(3,4). En una revisión de literatura realizada con 30 artículos entre el 2004 y 2014, se pudo evidenciar niveles variables entre el $40 \%$ y el $100 \%$ de contaminación bacteriana asociado a la presencia de Staphylococcus coagulasa negativo y Staphylococcus aureus como las bacterias más frecuentes y hasta en un 95,3\% resistentes a la meticilina(5).

El quirófano se presenta como un ambiente controlado en aspectos sépticos, convirtiéndose en el pilar fundamental de la atención segura hacia el paciente quirúrgico. La contaminación ambiental por muchos años no se consideró prioritaria a diferencias de múltiples factores que aumentan el riesgo de infección del sitio quirúrgico, pero la evidencia científica ha demostrado que controlar los factores del ambiente quirúrgico y una barrera aséptica hacia áreas no restringidas, desempeña un papel importante en la mitigación de la transmisión de microorganismos ${ }^{(6,7)}$.

Es importante resaltar que las superficies de los dispositivos móviles se encuentran fabricadas por plástico o metal, por su costo y durabilidad; las bacterias poseen una elevada capacidad de adherencia a materiales inertes, permitiendo la formación de biofilms y en muchos casos metabolizando el plástico y convirtiéndolo en nutrientes, como se ha reportado con $S$. aureus, Escherichia coli, Pseudomonas aeruginosa y $S$. epidermidis, esta última sobrevive largos periodos y es considerada una potencial fuente de infección ${ }^{(6,8)}$.

Estudios sobre la contaminación microbiana en dispositivos móviles, coinciden en que se debe generar mayor atención al aislamiento del $S$. aureus y en especial a las cepas multirresistentes y bacilos Gram negativos, por su posibilidad de resistir a condiciones de desecación y multiplicarse en ambientes cálidos como son las superficies de estos dispositivos ${ }^{(9)}$.

El riesgo generado por el uso no controlado de los dispositivos móviles en áreas restringidas y controladas asépticamente(10), plantea la necesidad de realizar este estudio en la institución involucrada, debido a la ausencia de restricciones para el uso, limpieza o desinfección de dispositivos móviles por parte del personal quirúrgico.

Es de esta manera que se plantea el objetivo de determinar la contaminación microbiana en dispositivos móviles del personal quirúrgico de una Institución de Salud de Pereira, Colombia en el primer semestre del año 2018, con el fin de crear estrategias que permitan disminuir el riesgo de contaminación cruzada asociada a su uso.

\section{Materiales y métodos}

Se realizó un estudio descriptivo, trasversal con los dispositivos móviles del personal quirúrgico de una institución hospitalaria de Pereira, constituido por 25 personas, entre ellas, cirujanos, médicos ayudantes, instrumentadores quirúrgicos, enfermeros y auxiliares. Se seleccionó al azar 10 personas que portaban celular, para realizar el análisis microbiológico a su dispositivo 
El criterio de selección de la institución participante se generó a partir de la aceptación de la misma a hacer parte en el proyecto de investigación y contar con personal que realizaba funciones dentro de las salas quirúrgicas (cirujano, médico ayudante, instrumentador, anestesiólogo y auxiliar de enfermería), en el momento de la toma de muestras. El trabajo de recolección consistió en una toma de muestras de superficie a 10 dispositivos móviles y sus estuches o cobertores mediante hisopo estéril a los cuales se les asignó un número del 1 al 10 para darle codificación durante el proceso.

Las muestras se transportaron en tubos de ensayo con agua peptonada al $1 \%$ hasta el momento del análisis, para el monitoreo microbiológico se tuvieron en cuenta 4 grupos de microorganismos analizados por técnica microbiológica recuento en placa profunda ${ }^{(11)}$ así: las muestras se incubaron en agar Plate Count para recuento total de mesófilos aerobios a $37^{\circ} \mathrm{C}$ x 24 horas, el recuento de coliformes totales y fecales se realizó en agar Brillance E. coli/Coliformes, y Petrifilm E. coli/Coliformes $37^{\circ} \mathrm{C}$ x 24 horas; los mohos y levaduras fueron sembrados en agar Sabouraud dextrosa $25^{\circ} \mathrm{C}$ x 5 días; todos los ensayos con controles microbiológicos previos. Estos procedimientos se realizaron en los laboratorios de ciencias básicas de la Fundación Universitaria De Área Andina.

Los controles microbiológicos de medios de cultivo se realizaron de la siguiente manera:

Control positivo: Cepas microbiológicas ATCC para verificar el funcionamiento de los medios. Staphylococcus aureus ATCC 25923 (mesófilos aerobios) recuento en placa profunda. Enterobacter aerogenes ATCC 13048 (coliformes totales) siembra por superficie Escherichia coli ATCC 25922 (coliformes fecales) Siembra por superficie Candida albicans (ATCC 60193), Aspergillus brasiliensis ATCC 16404 (mohos y levaduras) siembra por profundidad.

Los controles negativos de todos los medios se incubaron 48 horas después de preparados incluyendo la siembra en agar Plate Count para el agua peptonada, de esta última, un vial fue dejado para ser sembrado en al mismo momento del análisis de las muestras tomadas, corroborándose así que no estaba contaminada.
Para la toma de la muestra se utilizaron aplicadores desechables (hisopos) de plástico con punta de rayón estériles, empacados de manera individual, garantizando la inocuidad del procedimiento.

\section{Método de lectura}

Con respecto a la lectura e identificación de las bacterias, no se realizaron pruebas confirmatorias, ya que se empleó medio de cultivo selectivo para identificación de $E$. coli, y su diferenciación con coliformes totales.

Los datos fueron recolectados en Microsoft Excel $2015 \AA$, y se analizaron en SPSS v. 23. Para variables cualitativas se utilizaron frecuencias absolutas y relativas. Las variables cuantitativas fueron expresadas mediante medidas de tendencia central (Media) y desviación estándar (DE). Se realizó un análisis bivariado cualitativo utilizando el test Exacto de Fisher con un nivel se significancia $\leq 0,05$.

\section{Consideraciones éticas}

Esta investigación fue aprobada por el Comité de Investigación de la Facultad de Salud de la Fundación Universitaria del Área Andina, considerada como un estudio de riesgo mínimo según la Resolución № 008430 de 1993, que establece las normas científicas, técnicas y administrativas para la investigación en salud. También contó con la aprobación de la Institución de salud donde se realizó el estudio, y el suministro voluntario de los dispositivos móviles, posterior a la firma de consentimiento informado por parte del personal quirúrgico.

Todos los procedimientos microbiológicos se realizaron siguiendo las recomendaciones de la norma técnica NTC 4092 que establece los requisitos generales y directrices para análisis microbiológicos. Todo el personal que intervino en el estudio hizo un uso adecuado del equipo de bioseguridad, las muestras fueron procesadas dentro de la cabina de flujo laminar (debidamente calibrada) para garantizar la recuperación de los microorganismos propios de muestra, evitando la contaminación de los investigadores; una vez terminado el proceso los cultivos fueron autoclavados garantizando la eliminación adecuada del material.

\section{Resultados}

Se tomaron 5 dispositivos móviles de mujeres y 5 de hombres para evitar sesgos asociados a género. El 
40\% de las unidades de análisis eran de médicos cirujanos especialistas, el $20 \%$ de anestesiólogos, y un $40 \%$ de instrumentador quirúrgico, auxiliar de enfermería y médico residente.

Todas las muestras de la superficie de los dispositivos móviles y sus estuches o cobertores permitieron obtener cultivos exitosos, con microorganismos que pueden llegar a contribuir a infecciones cruzadas intrahospitalarias. El promedio de Unidades Formadoras de Colonias (UFC) por microorganismo fue de: 76,3 UFC/celular (DE=62) para mesófilos aerobios, 15,2 UFC/celular (DE=15,2) para mohos y levaduras y $7,1 \mathrm{UFC} /$ celular $(\mathrm{DE}=5,3$ ) para coliformes totales, el promedio reportado para coliformes totales fue inferior a $10 \mathrm{UFC} /$ celular en el total de muestras recolectadas. No se reportaron datos ausentes durante el procesamiento ni la recolección de los datos.

El elevado número de microorganismos mesófilos, de acuerdo a los límites de aceptabilidad de la resolución 1418 de 2011 del INVIMA (Instituto Nacional de Vigilancia de Medicamentos y Alimentos), puede considerarse de alto riesgo para las infecciones del sitio quirúrgico, relacionado a deficientes procesos de desinfección de dichos dispositivos.

El promedio ajustado de UFC, corregido tras la eliminación de valores extremos fue de 92,87 UFC/celular DE $=12,8$, para mesófilos aerobios, 22,37 UFC/celular DE $=6,3$ para mohos y levaduras y 13,5 UFC/celular $\mathrm{DE}=5,5$ para coliformes totales, no se encontraron patrones de variación en lo reportado para coliformes fecales.

Al realizar el análisis de asociaciones estadística entre variables cualitativas en relacional género y la presencia de microorganismos no se encontró diferencias estadísticas significativas para ninguno de los microorganismos presentes asociado al género; aerobios mesófilos $(p=0,5)$, mohos y levaduras $(p=0,08)$. En la institución no existen protocolos que permitan mitigar el riesgo de contaminación cruzada producto de los dispositivos móviles ingresados a áreas controladas asépticamente, tampoco se encontraron restricciones para el uso de los mismos.

\section{Discusión}

Los resultados obtenidos por el equipo investigador reportaron presencia de al menos $70 \mathrm{UFC} /$ celular para mesófilos aerobios, datos que son concordantes con lo reportado en la literatura mundial (3-5).

Las infecciones asociadas a la atención en salud, se siguen presentando como un riesgo significativo de aumento de la morbimortalidad entre los pacientes y los diversos agentes etiológicos responsables de tales infecciones, los cuales varían de un hospital a otro y también en diferentes regiones geográficas(7). Los teléfonos móviles se han convertido en una parte inseparable de la asistencia médica y se ha demostrado que son importantes fómites potenciales para la transmisión de infecciones nosocomiales ${ }^{(4)}$.

En la actualidad se reconoce la importancia de los teléfonos celulares dado que sus ventajas se reflejan en la solución de problemas técnicos y laborales al igual que la facilidad en el establecimiento de la comunicación; en el sector salud han evidenciado facilidades notorias al momento de diagnosticar y enfocar los manejos de pacientes en diferentes escenarios, dicha condición ha generado la popularización de estos elementos en todos los niveles sociales, hoy se han reportado 6.900 millones de contratos de telefonía según datos de la $\operatorname{OMS}^{(12)}$, sin embargo estos no se encuentran exentos de riesgos, adicional a los peligros biológicos continúan las alarmas respecto al impacto negativo de dichos móviles en la salud general de la población(12).

En un estudio realizado por Pérez et al(13)., en 71 teléfonos celulares del personal médico, pudieron evidenciar estafilococos coagulasa negativa 50\%, Staphylococcus aureus 32,4\% y enterobacterias 4,2\%, cabe anotar que dentro de este último grupo de microorganismos se encuentran: Escherichia, Shigella, Salmonella, Klebsiella, Serratia, Hafnia, Proteus, Morganella, Providencia, Yersinia, Edwarsiella, Citrobacter y Erwinia, actinomicetos $4,2 \%$ y solo el $9,8 \%$ de dispositivos resultaron negativos, con resultados similares a los encontrados en nuestro estudio. El estudio de Pérez et al. señala la importancia de establecer una práctica rutinaria de limpieza del teléfono celular y concientizar a la población de mantener estos hábitos de higiene. De igual manera Ustun y Cihangiroglu en un estudio realizado en 179 muestras de cultivo positivo de teléfonos móviles, pudo evidenciar altos niveles de contaminación donde $17(9,5 \%)$ presentaron Staphylococcus aureus resistente a meticilina (SARM) y 20 (11,2\%) Escherichia coli productora de BLEE, que puede causar infecciones intrahospitalarias(14). 
Dorost et al (15)., en los resultados de su estudio realizado en un hospital de Irán indican que la contaminación bacteriana de los teléfonos utilizados por los grupos investigados fue menor en los dispositivos de pantalla táctil que en los dispositivos de teclado y la contaminación fue mayor en teléfonos celulares del personal del hospital que en visitantes. Los teléfonos celulares de las mujeres presentan niveles más altos de colonización. Los microorganismos dominantes en el personal del hospital fueron las especies de Enterobacteriaceae, y Bacillus, especialmente las bacterias gram positivas esporuladas y la coagulasa estafilocócica negativa, respectivamente.

Murgier et al., realizaron un estudio pre y pos desinfección de 52 celulares en personal que ingresó en el quirófano del departamento de cirugía ortopédica del Centro Hospitalario Universitario, Toulouse (Francia). Antes de la descontaminación, el número medio de UFC era de 258 por teléfono (rango, 0 -1.664), después de la descontaminación, fue de 127 (rango, 0-800) ( $p=0,0001) .49$ teléfonos celulares tenían UFC antes de la descontaminación (94\%) y 39 después $(75 \%)(p=0,02)$, en dicho estudio no se encontró asociación estadística con el género, similar al fenómeno presentado en nuestro estudio (16).

Pillet et al., pudieron evidenciar que más de un tercio de los teléfonos móviles muestreados estaban contaminados con ARN de virus presentes en los distintos entornos clínicos. El ARN del Rotavirus (RV) se recuperó en gran parte de los trabajadores de la salud, especialmente en los del personal pediátrico(17). De esta manera se evidencia que la magnitud del problema empieza a incrementarse en relación a la transmisión cruzada de microorganismos entre las distintas áreas hospitalarias, lo cual se puede favorecer por fómites como los dispositivos móviles.

Con la investigación realizada, el equipo de trabajo pudo darse cuenta del papel potencial de los dispositivos móviles como fuente de infecciones cruzadas intrahospitalarias, similar a otros resultados de publicaciones ${ }^{(3-5)}$, el uso de dispositivos móviles dentro de los quirófanos se encuentra sin regulación. La OMS en 2016 publicó las 16 recomendaciones para prevenir infecciones de sitio operatorio en colaboración con la revista LANCET donde postulaban medidas como la adecuada oxigenación, uso de películas adherentes impregnadas con antibiótico, control de la temperatura y adecuados cuidados postoperatorios(18), no obstante dentro de estas recomendaciones no se reportaron datos frente al uso de dispositivos móviles.

Respecto al problema del uso de teléfonos móviles y la contaminación cruzada intrahospitalaria Brady et al. a través de una revisión narrativa en el año 2009 enfatizan en medidas tales como la educación del personal en salud, mediciones seriadas de contaminación intrahospitalaria, realización de protocolos para control de las infecciones, al igual que la restricción del uso de teléfonos celulares en locaciones con necesidad de asepsia (quirófanos, unidades de cuidado intensivo); lineamientos que se consideran costo efectivos al momento de restringir infecciones cruzadas(19).

Este estudio contó con una adecuada representatividad de la muestra para el área de quirófano de la institución objeto de estudio, los resultados evidencian la necesidad de establecer medidas preventivas para prevenir las infecciones asociadas a la atención en salud. La selección de una institución de cuarto nivel de complejidad en la ciudad de Pereira con impacto local y regional adiciona peso a las conclusiones al tratarse de un referente de importancia para la ciudad y la región. Pese a las fortalezas descritas existen limitaciones que deben ser consideradas al momento de interpretar los resultados, al tratarse de un estudio descriptivo de corte trasversal no es posible estructurar asociaciones causales respecto a la presencia de los microorganismos caracterizados y la presencia de móviles en quirófano, no obstante de acuerdo a los criterios de Hill(20) se evidencia que cumple con la secuencia temporal, plausibilidad biológica, evidencia experimental y consistencia hechos que permiten aproximar asociaciones causales estadísticas en futuros estudios.

\section{Conclusiones}

Existe una alta prevalencia de microorganismos presentes en los dispositivos móviles en la muestra de personal quirúrgico de una institución de Salud del municipio de Pereira, el cual conlleva un riesgo significativo asociado a las IAAS, por ello se recomienda tener controles exhaustivos de desinfección o de restricción a su uso dentro de las áreas quirúrgicas. 


\section{Recomendaciones}

Este estudio permite abrir la perspectiva a tal vez una fuente ignorada de infección, debido al continuo y casi obligatorio uso de dichos dispositivos, por el cual se recomienda establecer principios asepticos relacionado al uso de telefonos moviles.

Los resultados evidencian la presencia de microorganismos potencialmente patógenos (E. coli, Klebsiella, Proteus, Enterobacter, Citrobacter) en la superficie de los teléfonos móviles en un área hospitalaria donde se deben extremar las medidas asépticas, nuevas investigaciones son requeridas para evaluar factores de riesgo asociados, variables confusoras e interactoras al igual que la valoración de la fuerza de asociación entre la presencia de microorganismos en lo dispositivo móviles y la presencia de contaminación cruzada en los quirófanos, que según otros estudios es evidenciable por los que se recomiendan nuevos estudios multicéntricos que permitan realizar correlaciones entre los microorganismos presenten en los dispositivos móviles y las infecciones de heridas quirúrgicas.

\section{Conflicto de intereses}

Los autores declaran no poseer ningún conflicto de interés.

\section{Referencias}

1. Selim HS, Abaza AF. Microbial contamination of mobile phones in a health care setting in Alexandria, Egypt. GMS Hyg Infect Control [Internet]. 2015;10:1-9. Disponible en: https://www.ncbi.nlm.nih.gov/pubmed/25699226

2. Banawas S, Abdel-Hadi A, Alaidarous M, Alshehri B, Bin Dukhyil AA, Alsaweed M, et al. Multidrug-resistant bacteria associated with cell phones of healthcare professionals in selected hospitals in Saudi Arabia. Can J Infect Dis Med Microbiol [Internet]. 2018:1-7. Disponible en: https://www.ncbi.nlm.nih.gov/pmc/articles/PMC6323467

3. Katsuse Kanayama A, Takahashi H, Yoshizawa S, Tateda K, Kaneko A, Kobayashi I. Staphylococcus aureus surface contamination of mobile phones and presence of genetically identical strains on the hands of nursing personnel. Am J Infect Control [Internet]. 2017;45(8):929-31. Disponible en: http://dx.doi.org/10.1016/j.ajic.2017.02.011

4. Bodena D, Teklemariam Z, Balakrishnan S, Tesfa T. Bacterial contamination of mobile phones of health professionals in Eastern Ethiopia: antimicrobial susceptibility and associated factors. Trop Med Health [Internet]. 2019;47(15):1-10. Disponible en: https://doi.org/10.1186/s41182-019-0144-y

5. Morvai J, Szabó R. A mobil kommunikációs eszközök szerepe a fertőzések átvitelében. Szisztematikus irodalomelemzés. Orv Hetil [Internet]. 2015;156(20):802-7. Disponible en: http://www.akademiai.com/doi/abs/10.1556/650.2015.30 147
6. Hernández-orozco HG, Castañeda-narváez JL, Garza EA. Celulares y riesgo de infecciones intrahospitalarias. Infectol Pediatr [Internet]. 2017;30(2):45-7. Disponible en: http://www.medigraphic.com/pdfs/infectologia/lip2017/lip172a.pdf

7. Leaper DJ, Edmiston CE. Global guidelines for the prevention of surgical site infection [Internet]. World Health Organization. 2016. Disponible en: http://linkinghub.elsevier.com/retrieve/pii/S01956701163 05874

8. Debnath T, Bhowmik S, Islam T, Hassan Chowdhury MM. Presence of Multidrug-Resistant Bacteria on Mobile Phones of Healthcare Workers Accelerates the Spread of Nosocomial Infection and Regarded as a Threat to Public Health in Bangladesh. J Microsc Ultrastruct [Internet]. 2018;6(3):1659. Disponible en: https://www.ncbi.nlm.nih.gov/pubmed/30221143

9. Paz-Montes A, Fuenmayor-Boscán A, Sandrea L, Colmenares J, Marín M, Rodríguez E. Riesgo microbiológico asociado al uso de teléfonos móviles en laboratorios clínicos hospitalarios de Maracaibo-Venezuela. Kasmera [Internet]. 2015;43(2):148-57. Disponible en: http://www.scielo.org.ve/pdf/km/v43n2/art07.pdf

10. Pérez Castro JA, Castillo Vázquez CM, Rueda Rodríguez A, Jiménez Díaz IB, Domínguez de la Peña MA. Los pequeños grandes detalles de la seguridad del paciente. Rev CONAMED [Internet]. 2012;17(3):130-5. Disponible en: http://dialnet.unirioja.es/descarga/articulo/4062824.pdf\% 5Cnhttp://dialnet.unirioja.es/servlet/extart?codigo $=40628$ 24

11. Aquiahuatl R, Pérez C. Manual de prácticas de laboratorio de microbiología general. 2004. 119 p.

12. World Health Organisation (WHO). Campos electromagnéticos y salud pública: teléfonos móviles [Internet]. 2014. Disponible en: https://www.who.int/es/news-room/fact-

sheets/detail/electromagnetic-fields-and-public-healthmobile-phones

13. Pérez-Cano HJ, Reyes Santos MF, César Moreno BM. Microbiota in mobile phones of medical ophthalmologists. Arch Soc Esp Oftalmol [Internet]. 2019;94(2):55-9. Disponible en: https://doi.org/10.1016/j.oftale.2018.11.009

14. Ustun C, Cihangiroglu M. Health care workers' mobile phones: a potential cause of microbial cross-contamination between hospitals and community. J Occup Environ Hyg. 2012;9(9):538-42.

15. Dorost A, Safari Y, Akhlaghi M. Microbial contamination data of keypad and touch screen of cell phones among hospital and non-hospital staffs - A case study: Iran. Data Br [Internet]. 2018;20:80-4. Disponible en: http://dx.doi.org/10.1016/j.dib.2018.07.041

16. Murgier J, Coste J, Cavaignac E, Chiron $\mathrm{P}$, Bonnevialle $\mathrm{P}$, Laffosse J. Microbial flora on cell-phones in an orthopedic surgery room before and after decontamination. Orthop Traumatol Surg Res [Internet]. 2016;102(8):1093-6. Disponible http://dx.doi.org/10.1016/j.otsr.2016.09.014

17. Pillet S, Berthelot P, Mory O, Gay C, Viallon A, Lucht F, et al. Contamination of healthcare workers ' mobile phones by epidemic viruses. Clin Microbiol Infect [Internet]. 2016;22(5):456.e1-456.e6. Disponible en: http://dx.doi.org/10.1016/j.cmi.2015.12.008

18. World Health Organisation (WHO). WHO recommends 29 ways to stop surgical infections and avoid superbugs 
[Internet]. 2016. Disponible en: http://www.who.int/newsroom/detail/03-11-2016-who-recommends-29-ways-tostop-surgical-infections-and-avoid-superbugs

19. Brady RRW, Verran J, Damani NN, Gibb AP. Review of mobile communication devices as potential reservoirs of nosocomial pathogens. J Hosp Infect [Internet]. 2009;71(4):295-300. Disponible en: http://dx.doi.org/10.1016/j.jhin.2008.12.009
20. Sánchez FP. La búsqueda de las relaciones causales: el desafío del ejercicio diario de un epidemiólogo. Rev Medica Risaralda [Internet]. 2012;18(2):165-71. Disponible en: http://revistas.utp.edu.co/index.php/revistamedica/article /view/7913/4809 